\title{
EXISTENCE OF MONOTONIC ASYMPTOTICALLY CONSTANT SOLUTIONS FOR SECOND ORDER DIFFERENTIAL EQUATIONS
}

\author{
CRISTÓBAL GONZÁLEZ and ANTONIO JIMÉNEZ-MELADO \\ Dept. Análisis Matemático, Fac. Ciencias, Univ. Málaga, 29071 Málaga, Spain \\ e-mails:cmge@uma.es,melado@uma.es
}

(Received 11 January, 2007; revised 24 April, 2007; accepted 24 May, 2007)

\begin{abstract}
Starting from results of Dubé and Mingarelli, Wahlén, and Ehrström, who give conditions that ensure the existence and uniqueness of nonnegative nondecreasing solutions asymptotically constant of the equation

$$
y^{\prime \prime}(x)=-F\left(x, y(x), y^{\prime}(x)\right), \quad x \geq 0,
$$

we have been able to reduce their hypotheses in order to obtain the same existence results, at the expense of losing the uniqueness part. The main tool they used is the Banach Fixed Point Theorem, while ours has been the Schauder Fixed Point Theorem together with one version of the Arzelà-Ascoli Theorem.
\end{abstract}

2000 Mathematics Subject Classification. 34A12, 34A34, 34C10.

1. Introduction. In the present paper we consider the following differential equation in its general form

$$
y^{\prime \prime}(x)+F\left(x, y(x), y^{\prime}(x)\right)=0, \quad x \geq 0,
$$

where $F$ is a continuous function in its three variables (though the first result will assume that $F$ depends only on the first two variables). Our main interest is in giving sufficient conditions, 'simple enough', to ensure the existence of nonnegative nondecreasing, thus non oscillatory, solutions to equation (1).

Starting from results of Dubé and Mingarelli [2], Wahlén [6], and Ehrström [3], who give conditions that ensure the existence and uniqueness of nonnegative nondecreasing solutions asymptotically constant, we have been able to reduce their hypotheses in order to obtain the same existence results, at the expense of losing the uniqueness part. The main tool they used is the Banach Fixed Point Theorem, while ours, as other authors (see, e.g., $[\mathbf{1}, \mathbf{4}, \mathbf{5}, \mathbf{7}])$, has been the Schauder Fixed Point Theorem together with one version of the Arzelà-Ascoli Theorem.

The notation that we use throughout the paper is as follows: $\mathbb{R}^{+}=[0, \infty) \cdot \mathcal{C}\left(\mathbb{R}^{+}\right)$ is the space of bounded continuous real valued functions defined on $\mathbb{R}^{+}$. It is a Banach space when endowed with the sup norm $\|\cdot\|_{\infty}$. We may also write $\mathcal{C}\left(\mathbb{R}^{+}, J\right), J \subseteq \mathbb{R}$, for the subset of those $u \in \mathcal{C}\left(\mathbb{R}^{+}\right)$with values in $J$. Finally, $\mathcal{C}^{1}\left(\mathbb{R}^{+}\right)\left(\right.$or $\left.\mathcal{C}^{1}\left(\mathbb{R}^{+}, J\right)\right)$ is the space of bounded continuously differentiable real valued functions defined on $\mathbb{R}^{+}$with

Research partially supported by the Spanish (Grant MTM2004-00078) and regional Andalusian (Grant FQM210) Governments. 
bounded derivative. Again, it is a Banach space when endowed with the extended sup norm: $\|u\|_{\infty, 1} \equiv\|u\|_{\infty}+\left\|u^{\prime}\right\|_{\infty}$.

2. The results. Without further preliminaries, we present our first statement.

THEOREM 1. Consider the second order differential equation

$$
y^{\prime \prime}(x)+F(x, y(x))=0, \quad x \in \mathbb{R}^{+},
$$

together with the following assumptions:

(H1) $F \in \mathcal{C}\left(\mathbb{R}^{+} \times \mathbb{R}^{+}, \mathbb{R}^{+}\right)$.

(H2) There exist $M>0$ and $g: \mathbb{R}^{+} \rightarrow[0, M]$ with $g(x) \rightarrow 0$ as $x \rightarrow \infty$ such that

$$
\int_{x}^{\infty}(t-x) F(t, u(t)) d t \leq g(x), \quad x \in \mathbb{R}^{+},
$$

for all $u \in \mathcal{C}\left(\mathbb{R}^{+}\right)$with $M-g(x) \leq u(x) \leq M, \quad x \in \mathbb{R}^{+}$.

Then there exists a solution $y(x)$ to equation (2), with $y(x) \rightarrow M$ as $x \rightarrow \infty$, which is positive and nondecreasing on $(0, \infty)$ (strictly increasing unless there exists $x_{0} \geq 0$ such that $F(x, M) \equiv 0$ for all $\left.x \geq x_{0}\right)$.

Proof. First, we notice that it suffices to find a solution to the integral equation

$$
y(x)=M-\int_{x}^{\infty}(t-x) F(t, y(t)) d t, \quad x \in \mathbb{R}^{+},
$$

in the subset $C=\left\{u \in \mathcal{C}\left(\mathbb{R}^{+}\right): M-g(x) \leq u(x) \leq M, x \in \mathbb{R}^{+}\right\}$. Indeed, assume that $y \in C$ is a solution to the integral equation (3). Then it certainly satisfies the differential equation (2), is non-negative and has limit $M$ as $x \rightarrow \infty$ for it belongs to $C$ and we have $g(x) \rightarrow 0$ as $x \rightarrow \infty$. On the other hand, since $F$ is non-negative, we have by (2) that $y$ is a concave function on $\mathbb{R}^{+}$, which together with the non-negativity implies that $y$ is nondecreasing, even more, strictly increasing unless there is $x_{0} \geq 0$ defining the interval $\left[x_{0}, \infty\right)$ on which $y$ be constant. Since $y(x) \rightarrow M$ as $x \rightarrow \infty$, the possibility of being constant on a final interval implies that $y \equiv M$ on this interval. This is the case if and only if $F(x, M) \equiv 0$ for all $x \geq x_{0}$. Finally, all cases also yield that $y$ is strictly positive on $(0, \infty)$.

Before proceeding any further, let us extract more conclusions from the hypotheses (H1) and (H2). The first one comes from (H2) and is a uniform bound for the integral appearing in the integral equation (3), i.e., for all $x \geq 0$ and all $u \in C$,

$$
0 \leq \int_{x}^{\infty}(t-x) F(t, u(t)) d t \leq g(x) \leq M .
$$

Now the continuity of $F$ in (H1) tells us that $F$ is bounded in $[0,1] \times[0, M]$, say by $N$, which together with (H2), gives that for each $u \in C$, the function $F(t, u(t))$ is integrable on $\mathbb{R}^{+}$, even more, the integrals are uniformly bounded,

$$
\begin{aligned}
0 & \leq \int_{x}^{\infty} F(t, u(t)) d t \leq \int_{0}^{1} F(t, u(t)) d t+\int_{1}^{\infty} F(t, u(t)) d t \\
& \leq N+\int_{1}^{\infty} t F(t, u(t)) d t \leq N+\int_{0}^{\infty} t F(t, u(t)) d t \leq N+g(0) .
\end{aligned}
$$


Once these comments have been made, we look for a solution to the integral equation (3). This will be a fixed point of the operator

$$
T: C \rightarrow \mathcal{C}\left(\mathbb{R}^{+}\right), \quad T u(x)=M-\int_{x}^{\infty}(t-x) F(t, u(t)) d t,
$$

and the existence of such fixed point will be implied by the Schauder Fixed Point Theorem. Observe first that $C \subset \mathcal{C}\left(\mathbb{R}^{+}\right)$is a nonempty (the constant function equal to $M$ belongs to $C$ ), closed, convex and bounded subset of $\mathcal{C}\left(\mathbb{R}^{+}\right)$. Now, this operator is well defined and leaves invariant the set $C$. Indeed, by elementary calculus, if $u \in C$, $T u$ is more than continuous, it is continuously differentiable with derivative

$$
(T u)^{\prime}(x)=\int_{x}^{\infty} F(t, u(t)) d t, \quad x \geq 0,
$$

approaching 0 as $x \rightarrow \infty$, as (5) shows. The fact that $C$ is invariant under $T$ is obvious from (4).

It remains to prove that $T$ is compact on $C$. For this, it suffices to prove that $T$ is continuous on $C$ and that $T(C)$ is a relatively compact subset of $\mathcal{C}\left(\mathbb{R}^{+}\right)$.

We start showing that $T$ is continuous on $C$. Take $u_{0} \in C$ and $\varepsilon>0$. Since $g(x) \rightarrow 0$ as $x \rightarrow \infty$, there exists $x_{0}>0$ such that

$$
g(x) \leq \frac{\varepsilon}{4}, \quad \text { for all } x \geq x_{0} .
$$

Now, since $F$ is continuous on $\mathbb{R}^{+} \times \mathbb{R}^{+}$, it is uniformly continuous on $\left[0, x_{0}\right] \times[0, M]$, thus there exists $\delta>0$ such that if $y_{1}, y_{2} \in[0, M]$ and $\left|y_{1}-y_{2}\right| \leq \delta$ then

$$
\left|F\left(x, y_{1}\right)-F\left(x, y_{2}\right)\right| \leq \frac{\varepsilon}{x_{0}^{2}}, \quad \text { for all } x \in\left[0, x_{0}\right] .
$$

If now $u \in C$ and $\left\|u-u_{0}\right\|_{\infty} \leq \delta$ then, for $x \geq x_{0}$, using (6),

$$
\begin{aligned}
\left|T u(x)-T u_{0}(x)\right| & \leq \int_{x}^{\infty}(t-x)\left|F\left(t, u_{0}(t)\right)-F(t, u(t))\right| d t \\
& \leq \int_{x}^{\infty}(t-x) F\left(t, u_{0}(t)\right) d t+\int_{x}^{\infty}(t-x) F(t, u(t)) d t \leq 2 g(x) \leq \frac{\varepsilon}{2},
\end{aligned}
$$

and for $x \leq x_{0}$, using (6) and (7),

$$
\begin{aligned}
\left|T u(x)-T u_{0}(x)\right| & \leq\left(\int_{x}^{x_{0}}+\int_{x_{0}}^{\infty}\right)(t-x)\left|F(t, u(t))-F\left(t, u_{0}(t)\right)\right| d t \\
\ldots & \leq \int_{0}^{x_{0}} t \frac{\varepsilon}{x_{0}^{2}} d t+2 g\left(x_{0}\right)=\frac{\varepsilon}{x_{0}^{2}} \frac{x_{0}^{2}}{2}+2 g\left(x_{0}\right) \leq \varepsilon .
\end{aligned}
$$

Together this gives $\left\|T u-T u_{0}\right\|_{\infty} \leq \varepsilon$ whenever $u \in C$ and $\left\|u-u_{0}\right\|_{\infty} \leq \delta$, showing that $T$ is continuous at $u_{0}$.

We continue by proving that $T(C)$ is relatively compact. Observe that for each $x \in \mathbb{R}^{+}$, the set $T(C)(x) \equiv\{T u(x): u \in C\}$ is contained in $[0, M]$, so it is relatively compact in $\mathbb{R}^{+}$. Observe also that (5) implies that the set $\left\{(T u)^{\prime}: u \in C\right\}$ is (uniformly) bounded in $\mathcal{C}\left(\mathbb{R}^{+}\right)$by $N+g(0)$, and therefore $T(C)$ is uniformly Lipschitz in $\mathbb{R}^{+}$, providing that $T(C)$ is equicontinuous at each $x \in \mathbb{R}^{+}$. As a consequence, by the 
Arzelà-Ascoli Theorem, each sequence $\left\{T u_{n}\right\} \subset T(C) \subseteq C$ has a subsequence which converges uniformly on each compact subset of $\mathbb{R}^{+}$to a function $v \in C$.

But given the structure of the set $C$, a sequence $\left\{v_{n}\right\} \subset C$ which converges uniformly on each compact subset of $\mathbb{R}^{+}$to a function $v \in C$, must, in fact, converge uniformly to $v$ in $\mathbb{R}^{+}$. Indeed, take $\varepsilon>0$ and let $x_{1}>0$ be such that $g(x) \leq \varepsilon$ for all $x \geq x_{1}$. Since $v_{n}, n \in \mathbb{N}$, and $v$, all belong to $C$, their values remain between $M-g(x)$ and $M$, so

$$
\left|v_{n}(x)-v(x)\right| \leq g(x), \quad \text { all } n \in \mathbb{N} \text { and all } x \in \mathbb{R}^{+} .
$$

In particular,

$$
\left|v_{n}(x)-v(x)\right| \leq g(x) \leq \varepsilon, \quad \text { all } n \in \mathbb{N} \text { and all } x \geq x_{1} .
$$

Now, since $\left\{v_{n}\right\}$ converges uniformly to $v$ in $\left[0, x_{1}\right]$, there exists $n_{0} \in \mathbb{N}$ such that

$$
\left|v_{n}(x)-v(x)\right| \leq \varepsilon, \quad \text { all } n \geq n_{0} \text { and all } x \in\left[0, x_{1}\right] .
$$

This tells us that if $n \geq n_{0}$, then $\left\|v_{n}-v\right\|_{\infty} \leq \varepsilon$, proving that the convergence of $\left\{v_{n}\right\}$ to $v$ is uniform in $\mathbb{R}^{+}$.

With this observation we conclude that $T(C)$ is relatively compact in $\mathcal{C}\left(\mathbb{R}^{+}\right)$. And therefore, by the Schauder Fixed Point Theorem, the operator $T$ has a fixed point, which is one solution to our integral equation (3).

REMARK 1. A closer look at the proof of the theorem shows that one can replace $F \in \mathcal{C}\left(\mathbb{R}^{+} \times \mathbb{R}^{+}, \mathbb{R}^{+}\right)$by $F \in \mathcal{C}\left(\mathbb{R}^{+} \times[0, M], \mathbb{R}^{+}\right)$.

REMARK 2. The function $g(x)$ could become an important tool if one desires to know a bit more about the growth of the solutions to their limit $M$. The fact that the solution found is in the set $C$ tells us that it is always bounded below by the function $M-g(x)$.

Dubé and Mingarelli [2], and Wahlén [6] considered the same equation (2) and obtained a result on existence and uniqueness of positive non-decreasing solutions asymptotically equal to $M$. They assumed a Lipschitz condition on the second variable of $F$, which gave way to the use of the Banach fixed point theorem. The complete list of conditions they imposed are

(L1) $F \in \mathcal{C}\left(\mathbb{R}^{+} \times \mathbb{R}^{+}, \mathbb{R}^{+}\right)$.

(L2) $\int_{0}^{\infty} t F(t, u(t)) d t \leq M$ for all $u \in \mathcal{C}\left(\mathbb{R}^{+},[0, M]\right)$.

(L3) There exists $k \in \mathcal{C}\left(\mathbb{R}^{+}, \mathbb{R}^{+}\right)$with $\int_{0}^{\infty} t k(t) d t<\infty$ (Dubé and Mingarelli used the more restrictive hypothesis $\left.\int_{0}^{\infty} t k(t) d t<1\right)$ such that for any $u_{1}, u_{2} \in[0, M]$,

$$
\left|F\left(x, u_{1}\right)-F\left(x, u_{2}\right)\right| \leq k(x)\left|u_{1}-u_{2}\right|, \quad x \geq 0 .
$$

One could think that condition (L2) is weaker than (H2), because there is no need to find the function $g$. In fact, let us see that conditions (L1-3), all together, imply conditions (H1-2), showing thus that our result is an improvement on theirs, at least in what to the existence matters.

- (L1-3) $\Longrightarrow(\mathrm{H} 1-2)$. Take $u_{0} \in \mathcal{C}\left(\mathbb{R}^{+},[0, M]\right)$ and consider the function $g_{0}(x)=$ $\int_{x}^{\infty}(t-x) F\left(t, u_{0}(t)\right) d t, x \geq 0$, which is non-negative and bounded above by the constant $M$ by (L2), and also decreases to 0 at $\infty$. Now, for any $u \in \mathcal{C}\left(\mathbb{R}^{+},[0, M]\right)$ we have, by (L2), $\int_{x}^{\infty}(t-x) F(t, u(t)) d t \leq M$ for all $x \geq 0$; also, using that $\left\|u-u_{0}\right\|_{\infty} \leq M$ 
and (L3), we have for $x \geq 0$,

$$
\begin{aligned}
\int_{x}^{\infty}(t-x) F(t, u(t)) d t & \leq \int_{x}^{\infty}(t-x) F\left(t, u_{0}(t)\right) d t+\int_{x}^{\infty} t\left|F(t, u(t))-F\left(t, u_{0}(t)\right)\right| d t \\
& \leq g_{0}(x)+\int_{x}^{\infty} t k(t)\left|u(t)-u_{0}(t)\right| d t \leq g_{0}(x)+M \int_{x}^{\infty} t k(t) d t .
\end{aligned}
$$

If we now define, for $x \geq 0$,

$$
g(x)=\min \left\{g_{0}(x)+M \int_{x}^{\infty} t k(t) d t, M\right\},
$$

we observe that $g(x) \in[0, M]$ for all $x \geq 0$, that $g(x) \rightarrow 0$ as $x \rightarrow \infty$ and that

$$
\int_{x}^{\infty}(t-x) F(t, u(t)) d t \leq g(x), \quad x \geq 0,
$$

for all $u \in \mathcal{C}\left(\mathbb{R}^{+},[0, M]\right)$, in particular, for all $u \in \mathcal{C}\left(\mathbb{R}^{+}\right)$with $M-g(x) \leq u(x) \leq$ $M, x \geq 0$.

REMARK 3. Notice also that the existence of the function $g$ makes smaller the set of functions $u$ where one has to test the uniform integrability condition (H2) on functions of the type $(t-x) F(t, u(t))$. In order to make that set of test functions a little bit smaller, one could equally assume

$(\mathrm{H} 2)^{\prime}$ There exist $M>0$ and $g: \mathbb{R}^{+} \rightarrow[0, M]$ with $g(x) \rightarrow 0$ as $x \rightarrow \infty$ such that

$$
\int_{x}^{\infty}(t-x) F(t, u(t)) d t \leq g(x), \quad x \in \mathbb{R}^{+},
$$

for all increasing and concave $u \in \mathcal{C}\left(\mathbb{R}^{+}\right)$with $M-g(x) \leq u(x) \leq M, \quad x \in \mathbb{R}^{+}$. The proof of Theorem 1 now with (H2) replaced by (H2)' goes exactly the same as before.

The proof of Theorem 1 relied strongly on the fact that the hypotheses (H1-2) implied that $\int_{0}^{\infty} F(t, u(t)) d t$ is uniformly bounded for all $u \in C$. Our next Theorem includes dependance on the first derivative and the uniform boundedness of the integral of $F$ is not a straightforward deduction of the corresponding hypotheses (H1-2).

TheOrem 2. Assume that $M>0$, that $N \geq 0$, and that $F=F(x, y, z)$ is a nonnegative continuous function on $\mathbb{R}^{+} \times[0, M] \times[0, N]$, for which the following hypothesis is fulfilled.

(H3) There exist $g: \mathbb{R}^{+} \rightarrow[0, M]$ with $g(x) \rightarrow 0$ as $x \rightarrow \infty$ and $h: \mathbb{R}^{+} \rightarrow[0, N]$ with $h(x) \rightarrow 0$ as $x \rightarrow \infty$ such that

$$
\begin{gathered}
\int_{x}^{\infty}(t-x) F\left(t, u(t), u^{\prime}(t)\right) d t \leq g(x), \quad x \in \mathbb{R}^{+}, \\
\int_{x}^{\infty} F\left(t, u(t), u^{\prime}(t)\right) d t \leq h(x), \quad x \in \mathbb{R}^{+},
\end{gathered}
$$

for all $u \in C_{1}$, where $C_{1}$ is the set of concave and nondecreasing functions $u \in$ $\mathcal{C}^{1}\left(\mathbb{R}^{+},[0, M]\right)$ with $M-g(x) \leq u(x) \leq M$ and $0 \leq u^{\prime}(x) \leq h(x)$. 
Then there exists a solution $y(x)$ to the equation

$$
y^{\prime \prime}(x)+F\left(x, y(x), y^{\prime}(x)\right)=0,
$$

with $y(x) \rightarrow M$ as $x \rightarrow \infty$, which is positive, concave and nondecreasing on $(0, \infty)$.

Proof. Again, it suffices to find a fixed point to the operator $T$ in the set $C_{1}$ (closed, convex, bounded and not empty subset of $\mathcal{C}^{1}\left(\mathbb{R}^{+}\right)$), where this time, $T$ is defined as follows,

$$
T u(x)=M-\int_{x}^{\infty}(t-x) F\left(t, u(t), u^{\prime}(t)\right) d t, \quad x \in \mathbb{R}^{+}
$$

We let the reader check that $T$ is well defined and leaves invariant the set $C_{1}$. Note especially that, for $u \in C_{1}$ and $x \in \mathbb{R}^{+}$,

$$
(T u)^{\prime}(x)=\int_{x}^{\infty} F\left(t, u(t), u^{\prime}(t)\right) d t \leq h(x) \leq N
$$

Next we show that $T$ is continuous on $C_{1}$ (with respect to the $\|\cdot\|_{\infty, 1}$-topology). Take $u_{0} \in C_{1}$ and $\varepsilon>0$.

By $(\mathrm{H} 3)$, there exists $x_{0}>1$ such that

$$
g(x)<\frac{\varepsilon}{8}, \quad \text { and } \quad h(x)<\frac{\varepsilon}{8}, \quad \text { for all } x \geq x_{0} .
$$

Now, $F$ is uniformly continuous on $\left[0, x_{0}\right] \times[0, M] \times[0, N]$, so there exists $\delta>0$ such that

$$
\left|F\left(t_{1}, u_{1}, v_{1}\right)-F\left(t_{2}, u_{2}, v_{2}\right)\right|<\frac{\varepsilon}{4 x_{0}^{2}},
$$

whenever $\left(t_{j}, u_{j}, v_{j}\right) \in\left[0, x_{0}\right] \times[0, M] \times[0, N], j=1,2$, with $\left|t_{1}-t_{2}\right|+\left|u_{1}-u_{2}\right|+$ $\left|v_{1}-v_{2}\right|<\delta$.

Thus, if $u \in C_{1}$ with $\left\|u-u_{0}\right\|_{\infty, 1}<\delta$, and $x \leq x_{0}$, we have

$$
\begin{aligned}
\left|T u(x)-T u_{0}(x)\right| \leq & \int_{x}^{\infty}(t-x)\left|F\left(t, u(t), u^{\prime}(t)\right)-F\left(t, u_{0}(t), u_{0}^{\prime}(t)\right)\right| d t \\
\leq & \int_{x}^{x_{0}}(t-x)\left|F\left(t, u(t), u^{\prime}(t)\right)-F\left(t, u_{0}(t), u_{0}^{\prime}(t)\right)\right| d t \\
& +\int_{x_{0}}^{\infty}(t-x)\left(F\left(t, u(t), u^{\prime}(t)\right)+F\left(t, u_{0}(t), u_{0}^{\prime}(t)\right)\right) d t \\
\leq & \frac{\varepsilon}{4 x_{0}^{2}} \frac{x_{0}^{2}}{2}+2 g\left(x_{0}\right)<\frac{\varepsilon}{2},
\end{aligned}
$$


and

$$
\begin{aligned}
\left|(T u)^{\prime}(x)-\left(T u_{0}\right)^{\prime}(x)\right| \leq & \int_{x}^{\infty}\left|F\left(t, u(t), u^{\prime}(t)\right)-F\left(t, u_{0}(t), u_{0}^{\prime}(t)\right)\right| d t \\
\leq & \int_{x}^{x_{0}}\left|F\left(t, u(t), u^{\prime}(t)\right)-F\left(t, u_{0}(t), u_{0}^{\prime}(t)\right)\right| d t \\
& +\int_{x_{0}}^{\infty}\left(F\left(t, u(t), u^{\prime}(t)\right)+F\left(t, u_{0}(t), u_{0}^{\prime}(t)\right)\right) d t \\
\leq & \frac{\varepsilon}{4 x_{0}^{2}} x_{0}+2 h\left(x_{0}\right)<\frac{\varepsilon}{2} .
\end{aligned}
$$

Of course, if $x>x_{0}$, only the second summands in the above estimates are needed to obtain

$$
\left|T u(x)-T u_{0}(x)\right| \leq 2 g(x)<\frac{\varepsilon}{4}, \quad \text { and } \quad\left|(T u)^{\prime}(x)-\left(T u_{0}\right)^{\prime}(x)\right| \leq 2 h(x)<\frac{\varepsilon}{4} .
$$

All this yields $\left\|T u-T u_{0}\right\|_{\infty, 1}<\varepsilon$ whenever $u \in C_{1}$ with $\left\|u-u_{0}\right\|_{\infty, 1}<\delta$.

Next, we shall prove that $T C_{1}=\left\{T u: u \in C_{1}\right\}$ is relatively compact in $\mathcal{C}^{1}\left(\mathbb{R}^{+}\right)$, by applying again the Arzelà-Ascoli Theorem (twice) on $T C_{1}$ and $\left(T C_{1}\right)^{\prime}=\left\{(T u)^{\prime}\right.$ : $\left.u \in C_{1}\right\}$.

Notice that for a given $x \in \mathbb{R}^{+}$, we have

$$
T C_{1}(x) \subseteq[0, M], \quad \text { and } \quad\left(T C_{1}\right)^{\prime}(x) \subseteq[0, N],
$$

so both sets are relatively compact in $\mathbb{R}^{+}$.

Since $\left(T C_{1}\right)^{\prime}$ is (uniformly) bounded as a set of $\mathcal{C}\left(\mathbb{R}^{+}\right)$, then $T C_{1}$ is uniformly Lipschitz in $\mathbb{R}^{+}$, and consequently, $T C_{1}$ is equicontinuous at each $x \in \mathbb{R}^{+}$.

Also, notice that the set of second derivatives $\left(T C_{1}\right)^{\prime \prime}=\left\{(T u)^{\prime \prime}: u \in C_{1}\right\}$ is well defined and coincides with the set of functions $\left\{F\left(\cdot, u(\cdot), u^{\prime}(\cdot)\right): u \in C_{1}\right\}$, which is locally uniformly bounded in $\mathbb{R}^{+}$, i.e., for each $x_{0} \in \mathbb{R}^{+}$there exists a neighbourhood $U_{x_{0}}$ of $x_{0}$ in $\mathbb{R}^{+}$and a constant $L\left(x_{0}\right)$ such that $0 \leq F\left(x, u(x), u^{\prime}(x)\right) \leq L\left(x_{0}\right)$ for all $x \in$ $U_{x_{0}}$ (for instance, $U_{x_{0}}=\left[0, x_{0}+1\right)$ and $L\left(x_{0}\right)=\max \left\{F(x, u, v):(x, u, v) \in\left[0, x_{0}+\right.\right.$ $1] \times[0, M] \times[0, N]\})$. This implies that $\left(T C_{1}\right)^{\prime}$ is locally uniformly Lipschitz in $\mathbb{R}^{+}$ and, consequently, that $\left(T C_{1}\right)^{\prime}$ is equicontinuous at each $x \in \mathbb{R}^{+}$.

All this implies (by applying twice the Arzelà-Ascoli Theorem) that each sequence $\left\{T u_{n}\right\} \subset T C_{1}$ contains a subsequence $\left\{T u_{n_{k}}\right\}$ such that both sequences, $\left\{T u_{n_{k}}\right\}$ and $\left\{\left(T u_{n_{k}}\right)^{\prime}\right\}$, converge uniformly on each compact subset of $\mathbb{R}^{+}$to given functions $\tilde{u}$ and $\tilde{v}$. An argument similar to the one given at the end of the proof of Theorem 1 gives that, in fact, the convergence is uniform in all $\mathbb{R}^{+}$for both subsequences. The completeness of $C_{1}$ as a closed subset of $\mathcal{C}^{1}\left(\mathbb{R}^{+}\right)$implies that $\tilde{u}^{\prime}=\tilde{v}$ and that $T u_{n_{k}} \rightarrow \tilde{u}$ in the $\|\cdot\|_{\infty, 1}$-norm.

With this we conclude that $T C_{1}$ is relatively compact in $\mathcal{C}^{1}\left(\mathbb{R}^{+}\right)$. And therefore, by the Schauder Fixed Point Theorem, the operator $T$ has a fixed point, which is one solution to our integral equation.

In a recent paper, Ehrnström [3] has considered the same equation of Theorem 2, and has given a result about uniqueness and existence of solutions asymptotically equal to $M$, using hypotheses similar to the ones given by Wahlén [6], i.e., imposing a Lipschitz condition on the second and third variables of $F$. All these conditions are listed below, besides the non-negativity and continuity of $F$. 
(L4) $\int_{0}^{\infty} t F\left(t, u(t), u^{\prime}(t)\right) d t \leq M$ for all $u \in \mathcal{C}^{1}\left(\mathbb{R}^{+},[0, M]\right)$ with $u^{\prime} \geq 0$.

(L5) There exists $k \in \mathcal{C}\left(\mathbb{R}^{+}, \mathbb{R}^{+}\right)$with $\int_{0}^{\infty} t k(t) d t<\infty$ such that for any $u_{1}, u_{2} \in$ $[0, M]$, and any $v_{1}, v_{2} \in \mathbb{R}^{+}$,

$$
\left|F\left(x, u_{1}, v_{1}\right)-F\left(x, u_{2}, v_{2}\right)\right| \leq k(x)\left(\left|u_{1}-u_{2}\right|+\left|v_{1}-v_{2}\right|\right), \quad x \geq 0 .
$$

Again, as we did before, we prove that our result includes the one of Ehrnström, except for the uniqueness part. For that, assume (L4-5), and take $u \in \mathcal{C}^{1}\left(\mathbb{R}^{+},[0, M]\right)$ concave and nondecreasing. Set $K(x)=\int_{x}^{\infty} k(t) d t, K_{\infty}=\max \{k(x): x \in[0,1]\}$, and $A(x)=\int_{x}^{\infty} F(t, M, 0) d t$. These quantities exist, are nonnegative and finite, and $K(x)+$ $A(x) \searrow 0$ as $x \nearrow \infty$. Observe that the concavity and monotonicity of $u$ easily implies that $x u^{\prime}(x) \leq u(x)-u(0)(\leq M)$ for all $x \in \mathbb{R}^{+}$. Then, using the Lipschitz condition, for $0 \leq x<1$,

$$
\begin{aligned}
\int_{x}^{\infty} F\left(t, u(t), u^{\prime}(t)\right) d t & \leq \int_{x}^{\infty}\left|F\left(t, u(t), u^{\prime}(t)\right)-F(t, M, 0)\right| d t+\int_{x}^{\infty} F(t, M, 0) d t \\
& \leq \int_{x}^{\infty} k(t)\left(|u(t)-M|+u^{\prime}(t)\right) d t+A(x) \\
& \leq M \int_{x}^{\infty} k(t) d t+\int_{x}^{1} k(t) u^{\prime}(t) d t+\int_{1}^{\infty} k(t) u^{\prime}(t) d t+A(x) \\
& \leq M K(x)+K_{\infty}(u(1)-u(x))+\int_{1}^{\infty} k(t) t u^{\prime}(t) d t+A(x) \\
& \leq M K(x)+M K_{\infty}+\int_{1}^{\infty} k(t)(u(t)-u(0)) d t+A(x) \\
& \leq M K(x)+M K_{\infty}+M K(1)+A(x) \leq N
\end{aligned}
$$

where $N=2 M K(0)+M K_{\infty}+A(0)$. And for $x>1$,

$$
\begin{aligned}
\int_{x}^{\infty} F\left(t, u(t), u^{\prime}(t)\right) d t & \leq \int_{x}^{\infty}\left|F\left(t, u(t), u^{\prime}(t)\right)-F(t, M, 0)\right| d t+\int_{x}^{\infty} F(t, M, 0) d t \\
& \leq \int_{x}^{\infty} k(t)\left(|u(t)-M|+u^{\prime}(t)\right) d t+A(x) \\
& \leq M \int_{x}^{\infty} k(t) d t+\int_{x}^{\infty} k(t) t u^{\prime}(t) d t+A(x) \\
& \leq 2 M K(x)+A(x) \leq N
\end{aligned}
$$

Define

$$
h(x)= \begin{cases}M K(x)+M K_{\infty}+M K(1)+A(x), & \text { if } 0 \leq x \leq 1, \\ 2 M K(x)+A(x), & \text { if } 1<x\end{cases}
$$

Observe that $h$ is independent of $u$, that $0 \leq h(x) \leq N$ for all $x \in \mathbb{R}^{+}$, and that $h(x) \rightarrow 0$ as $x \rightarrow \infty$. Now one has to find the appropriate $g$, with $0 \leq g(x) \leq M, x \in \mathbb{R}^{+}$, and $g(x) \rightarrow 0$ as $x \rightarrow \infty$. This is done by the same procedure as before, and is left to the reader. (For instance, setting $g_{0}(x)=\int_{x}^{\infty}(t-x) F(t, M, 0) d t$ and $K_{1}(x)=\int_{x}^{\infty} t k(t) d t$, then $g(x)=\min \left\{M,(M+N) K_{1}(x)+g_{0}(x)\right\}$.) 
EXAMPLE . To illustrate our result compared with the one of Ehrström, we consider the very simple linear equation $y^{\prime \prime}+y^{\prime}=0$. In this case $F(x, y, z)=z$. The solutions of this equation are of the type $y=A+B e^{-x}$. In particular, those nonnegative and nondecreasing solutions asymptotically equal to 1 are reached when $A=1$ and $B \in$ $[-1,0]$. Obviously then, the result of Ehrström does not apply because they are not unique. On the other hand, taking $g(x)=h(x)=e^{-x}, x \in \mathbb{R}^{+}$, we see that our result does apply: If $u \in \mathcal{C}^{1}\left(\mathbb{R}^{+}\right)$is a nonnegative and concave function with $1-g(x) \equiv$ $1-e^{-x} \leq u(x) \leq 1$ and $0 \leq u^{\prime}(x) \leq e^{-x} \equiv h(x)$, then, for $x \in \mathbb{R}^{+}$,

$$
\begin{aligned}
\int_{x}^{\infty}(t-x) u^{\prime}(t) d t & =-\left.(t-x)(1-u(t))\right|_{t=x} ^{\infty}+\int_{x}^{\infty}(1-u(t)) d t \\
& \leq \int_{x}^{\infty} e^{-t} d t=e^{-x}=g(x)
\end{aligned}
$$

and

$$
\int_{x}^{\infty} u^{\prime}(t) d t=1-u(x) \leq e^{-x}=h(x) .
$$

Acknowledgments. We are grateful to the referee for the careful reading of the manuscript, especially for making us re-consider condition $(\mathrm{H} 2)$ with the inequality presented there as is, instead of $\int_{x}^{\infty} t F(t, u(t)) d t \leq g(x), x \in \mathbb{R}^{+}$.

\section{REFERENCES}

1. A. Constantin, On the existence of positive solutions of second order differential equations, Ann. Mat. Pura Appl. (4) 184 (2005), no. 2, 131-138.

2. S. D. Dubé and A. B. Mingarelli, Note on a non-oscillation theorem of Atkinson, Electron. J. Differential Equations 2004 (2004), no. 22 1-6, available at http://ejde.math. txstate.edu.

3. M. Ehrnström, Positive solutions for second-order nonlinear differential equations, Nonlinear Anal. 64 (2006), 1608-1620.

4. O. Lipovan, On the asymptotic behaviour of the solutions to a class of second order nonlinear differential equations, Glasgow Math. J. 45 (2003), 179-187.

5. O. G. Mustafa and Y. V. Rogovchenko, Global existence of solutions with prescribed asymptotic behavior for second-order nonlinear differential equations, Nonlinear Anal. 51 (2002), 339-368.

6. E. Wahlén, Positive solutions of second-order differential equations, Nonlinear Anal. 58 (2004), 359-366.

7. Z. Yin, Monotone positive solutions of second-order nonlinear differential equations, Nonlinear Anal. 54 (2003), 391-403. 